\title{
Fermi Large Area Telescope observations of the Sun: The first ten years
}

\author{
Melissa Pesce-Rollins* \\ INFN-Pisa \\ E-mail: melissa.pesce.rollins@pi.infn.it
}

Nicola Omodei

Stanford University

E-mail: nicola.omodeiestanford.edu

\author{
Alice J. Allafort \\ Stanford University \\ E-mail: allafortestanford.edu
}

Vahè Petrosian

Stanford University

E-mail: vahepestanford.edu

\section{Silvia Rainò}

INFN-Bari

E-mail: silvia.raino@ba.infn.it

\section{Wei Liu}

Lockheed Martin Solar and Astrophysics Laboratory

E-mail: weiliuelmsal.com

The Fermi Large Area Telescope (LAT) observations of the active Sun provide the largest sample of detected solar flares with emission greater than $30 \mathrm{MeV}$ to date. These include detections of impulsive and sustained emission, extending up to 20 hours in the case of the 2012 March 7 $\mathrm{X}$-class flares. Of particular interest is the first detection of $>100 \mathrm{MeV}$ gamma-ray emission from three solar flares whose positions behind the limb were confirmed by the STEREO spacecrafts. The LAT data provides a new observational channel that, when combined with observations from across the electromagnetic spectrum, provide a unique opportunity to diagnose the mechanisms of high-energy emission and particle acceleration in solar flares. We will present an overview of these observations including the emission of the Sun in its quiescent state and discuss how these observations provide constrains on different emission mechanisms.

7th Fermi Symposium 2017

15-20 October 2017

Garmisch-Partenkirchen, Germany

\footnotetext{
*Speaker.
} 


\section{Introduction}

During its first nine and a half years in orbit, the Fermi Large Area Telescope (LAT) [1] has detected $>30 \mathrm{MeV}$ gamma-ray emission from more than 40 solar flares, nearly 10 times more than EGRET [2] on-board the Compton Gamma-Ray Observatory, GRS [3] on-board the Solar Maximum Mission (SMM) and CORONAS-F [4]. The Fermi detections sample both the impulsive [5] and the long-duration phases [6] including the longest extended emission ever detected ( 20 hours) from the 2012 March 7 GOES X-class flares [7]. In addition to the impulsive and long-duration solar flares, the LAT has also provided the first observations of $>100 \mathrm{MeV}$ emission from flares whose footpoints are occulted by the solar limb $[8,9]$. The LAT also observes the Sun during its quiescent phase and detected the two component gamma-ray emission for the first time [10]. In this talk I will cover some of the highlights of these observations.

\section{The quiescent Sun}

When the Sun is not flaring, it is a steady, faint source of gamma-rays. This emission consists of two components: a point-like emission from the solar disk originating from hadronic cosmic ray cascades in the solar atmosphere and an extended emission from the inverse Compton scattering of cosmic ray electrons on solar photons in the heliosphere. The point-like emission was first mentioned by [11] and [12] and the existence of an additional, spatially extended component was not realized until recently $[13,14]$. The quiet Sun was detected for the first time in the EGRET data [15]. Given that the gamma-ray emission from the quiescent Sun is due to galactic cosmic rays this emission is also expected to be anti-correlated with the solar cycle because of the change of the heliospheric flux of the Galactic cosmic rays. Such time variations have been detected in Fermi-LAT data [16].

Thanks to the increased sensitivity of the LAT with respect to previous gamma-ray space missions, these two components have been observed with high statistical significance with just the first 18 months of data [10]. Now that the solar cycle 24 is reaching it end it is possible to perform studies to observe the modulation of the flux of these two components as a function of time. The 9 year Pass 8 Clean class spectral energy distribution (SED) in the energy range from $80 \mathrm{MeV}$ to $80 \mathrm{GeV}$ of the solar disk component in different time intervals is shown in figure 1. The flux variation as a function of the solar cycle can be seen from this figure, where the higher/lower SED (red/blue points) corresponds to the minimum/maximum solar activity. This same anti-correlation is also seen for the Inverse Compton component. Further details on this study can be found in the poster presentations 212 (Time evolution and study of the two emission components from the Quiescent Sun in 9 years of Fermi-LAT data by Rainò et al.) and 121 (The Quiet Sun in Gamma Rays: Modeling Cosmic-Ray Electrons in the Inner Heliosphere by Orlando et al.) of this $7^{\text {th }}$ Fermi Symposium.

\section{The flaring Sun}

Magnetic reconnection is believed to be at the origin of particle acceleration in solar flares and the last few decades of hard X-ray observations by SMM [17], Yohkoh [18, 19] and RHESSI [20, 


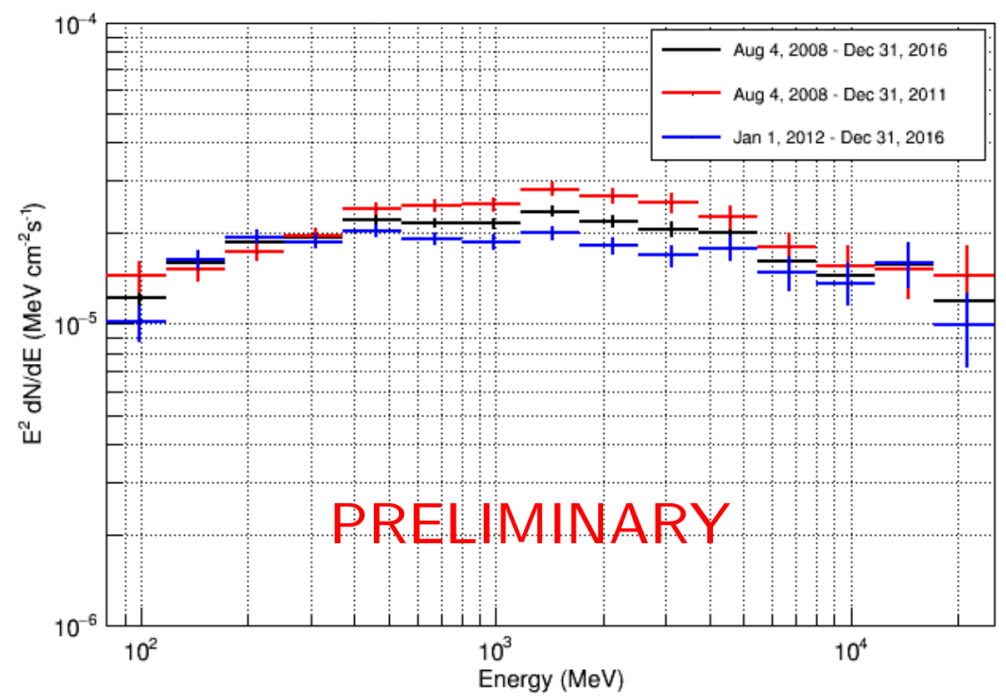

Figure 1: Spectral energy distribution (SED) of the solar disk component in different time intervals. The time intervals shown correspond to August 4, 2008 - December 31, 2016 (black), August 4, 2008 - December 31, 2011 (red) and January 1, 2012 - December 31, 2016 (blue).

21, 22] have provided support for this model. Gamma-ray emission from solar flares is produced by interactions of high-energy particles with ambient plasma through three different emission processes: bremsstrahlung (in the $10 \mathrm{keV}-100 \mathrm{MeV}$ energy range), nuclear de-excitation $(\sim 0.5-8$ $\mathrm{MeV}$ energy range) and pion decay $(>10 \mathrm{MeV})$. Prior to the launch of Fermi, the sample of solar flares with detected emission $>100 \mathrm{MeV}$ was rather small and limited to the brightest X-ray flares (GOES X class). Hour-long gamma-ray emission was observed in a few rare cases and the longest duration was the eight hour emission from the 1991 June $11^{\text {th }}$ flare [23].

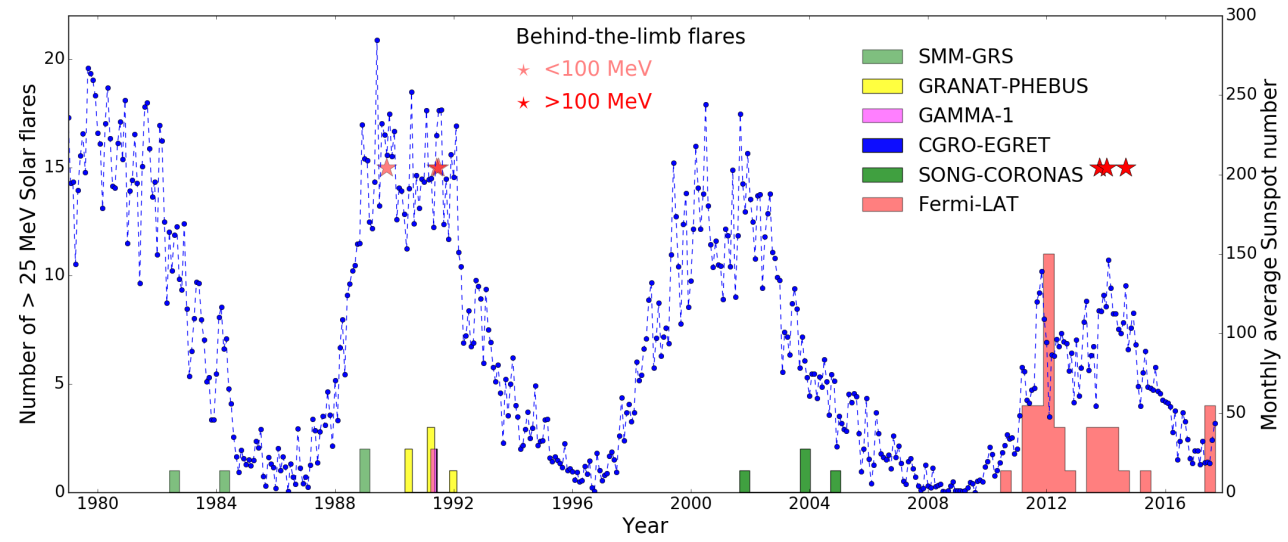

Figure 2: Number of solar flares with observed emission $>25 \mathrm{MeV}$ (left axis) together with the monthly averaged sunspot numbers (right axis) from 1980 to present. The light red stars represent the behind-thelimb solar flares detected with emission $<100 \mathrm{MeV}$ and the dark stars represent the behind-the-limb solar flares detected with emission $>100 \mathrm{MeV}$.

Fermi-LAT observations of the Sun have revealed 44 solar flares in the first nine and a half 
years of mission, increasing the overall sample of these high-energy flares by almost a factor of ten as illustrated in figure 2. More than half of these detections are from moderate GOES X-ray flares ( $\mathrm{M}$ class and one $\mathrm{C}$ class) and 20 have gamma-ray emission lasting more than 1 hour (with durations reaching up to almost 20 hours [7]). This result indicates that long-duration emission is fairly common (contrarily to what was believed before Fermi) and not necessarily related to bright X-ray flares. The Fermi-LAT detected solar flares distributed over the GOES X-ray class and the distribution of the duration (in hours) of the Fermi-LAT flares with emission lasting more than 1 hour are shown in figure 3. All of the long duration flares are associated with fast coronal mass ejections (CMEs) suggesting a potential relation between the flaring events and these shocks. Preliminary correlation studies show a correlation of 0.6 between the CME linear speed and the fluence of the long-duration (emission lasting more than one hour) gamma-ray flares whereas there is no apparent correlation between the $>100 \mathrm{MeV}$ fluence and the GOES peak X-ray flux (see figure 4). Another interesting aspect of the LAT observations of solar flares is that the emission centroid (for the brightest LAT flares) is localized on the active region from the flaring activity originated.
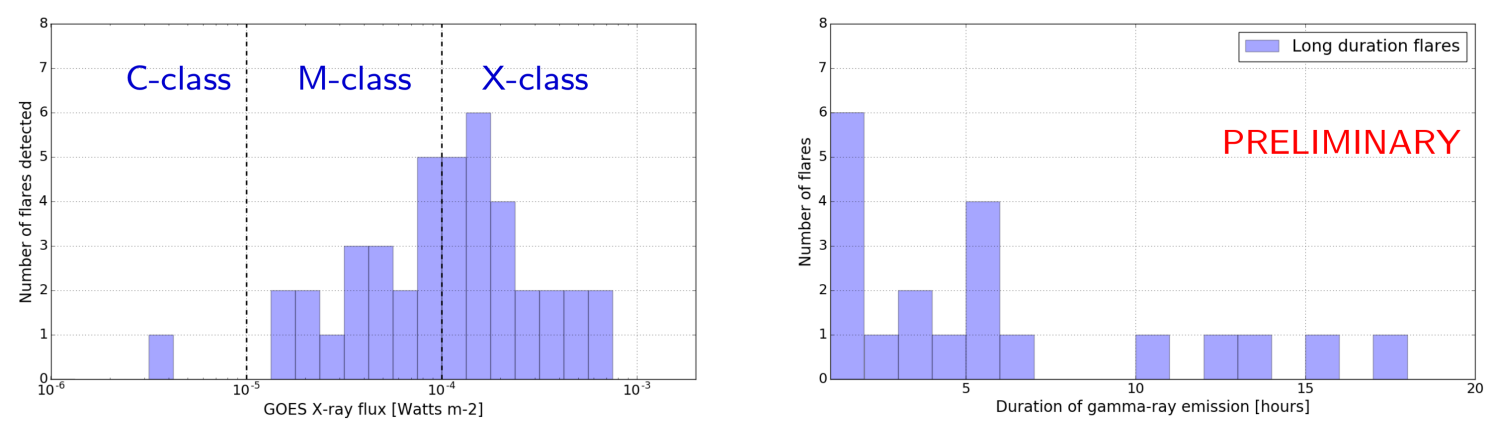

Figure 3: Fermi-LAT detected solar flares distributed over the GOES X-ray class (left panel) and the distribution of the duration (in hours) of the Fermi-LAT flares with emission lasting more than 1 hour (right panel).
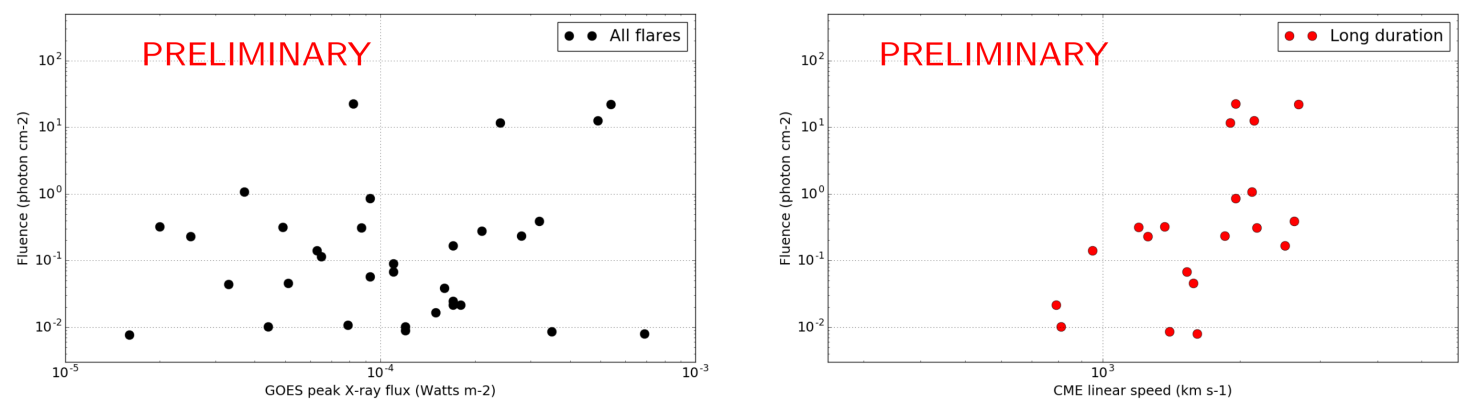

Figure 4: Correlation between the $>100 \mathrm{MeV}$ fluence of the Fermi-LAT detected flares versus the GOES peak X-ray flux (left panel) and the correlation between $>100 \mathrm{MeV}$ fluence versus the Coronal Mass Ejection (CME) linear speed (right panel). A Pearson's correlation of 0.24 is found for data sample in the left panel and 0.60 for the sample in the right panel. 


\subsection{Behind-The-Limb flares}

Three behind-the-limb (BTL) flares with emission up to $100 \mathrm{MeV}$ were observed during solar cycles 21 and 22 [24, 25, 26]. As mentioned in section 1, thanks to the LAT GeV emission has been detected by solar flares whose active region was behind the visible solar disk for the first time. Two of the BTL flares detected by the LAT originate from the eastern limb, the third from the western limb and their distances from the visible disk range from $10^{\circ}$ to $40^{\circ}$ [9]. These events are of particular interest because they provide a unique opportunity to diagnose the mechanisms of high-energy emission and particle acceleration in solar flares. This is because in the standard flare model involving magnetic reconnection, the accelerated particles precipitate to the solar surface to interact in a compact region near the active region from which the flaring activity originated. Furthermore, gamma-ray emission from pion decay processes require densities great than $10^{12} \mathrm{~cm}^{-3}$. Therefore these observations of gamma-rays from BTL flares imply that a spatially extended flare component must exist and that it can subtend a large range of heliolongitudes in order to allow the accelerated particles to reach and interact in the visible solar disk [27]. Another possibility is that the acceleration and emission occur in the Corona but this requires larger than usual Coronal densities. A possible spatial component could be the CME associated with the flare [28], the role of these shocks in the long-duration gamma-ray flares is supported by the correlation illustrated in the left panel of figure 4 .

\subsection{The September 2017 flares}

After almost an entire year of a nearly spotless Sun and no flaring activity, the largest flare (GOES class X9.3) of the solar cycle erupted from active region 2673 on September 6, 2017. This flare was very bright in gamma-rays and the emission detected by the LAT lasted for almost 15 hours (see the Astronomers Telegram (ATeL) 10720). Only 4 days later on September 10 an X8.2 flare erupted from the same active region and for 12 hours the Sun was the brightest gamma-ray source in the sky (see ATeL 10721 for further details). This flare was also associated with the second ground level enhancement (GLE 72) of this solar cycle. In figure 5 are the 12 hour intensity maps of the gamma-ray sky seen by Fermi-LAT before and during the September 10 solar flare. A paper on this flare is in preparation.

\section{Summary}

The Fermi-LAT has increased the number of observed high energy gamma-ray flares by almost a factor of ten during its first nine and a half years of mission. From this increased sample of flares we find that there are essentially three different classes of flares: impulsive, long-duration and behind-the-limb. The first two classes can be further divided into flares where only the impulsive/long duration emission was observed ${ }^{1}$ and flares where both the impulsive and long duration emission was observed. The differences between these flare types can provide valuable information on the underlying mechanisms driving these flares.

One possible scenario that emerges from these observations is that the impulsive emission can be explained in the standard model where magnetic reconnection is responsible for accelerating

\footnotetext{
${ }^{1}$ Even if the Sun was in the field of view of the LAT, no impulsive/long-duration emission was detected.
} 


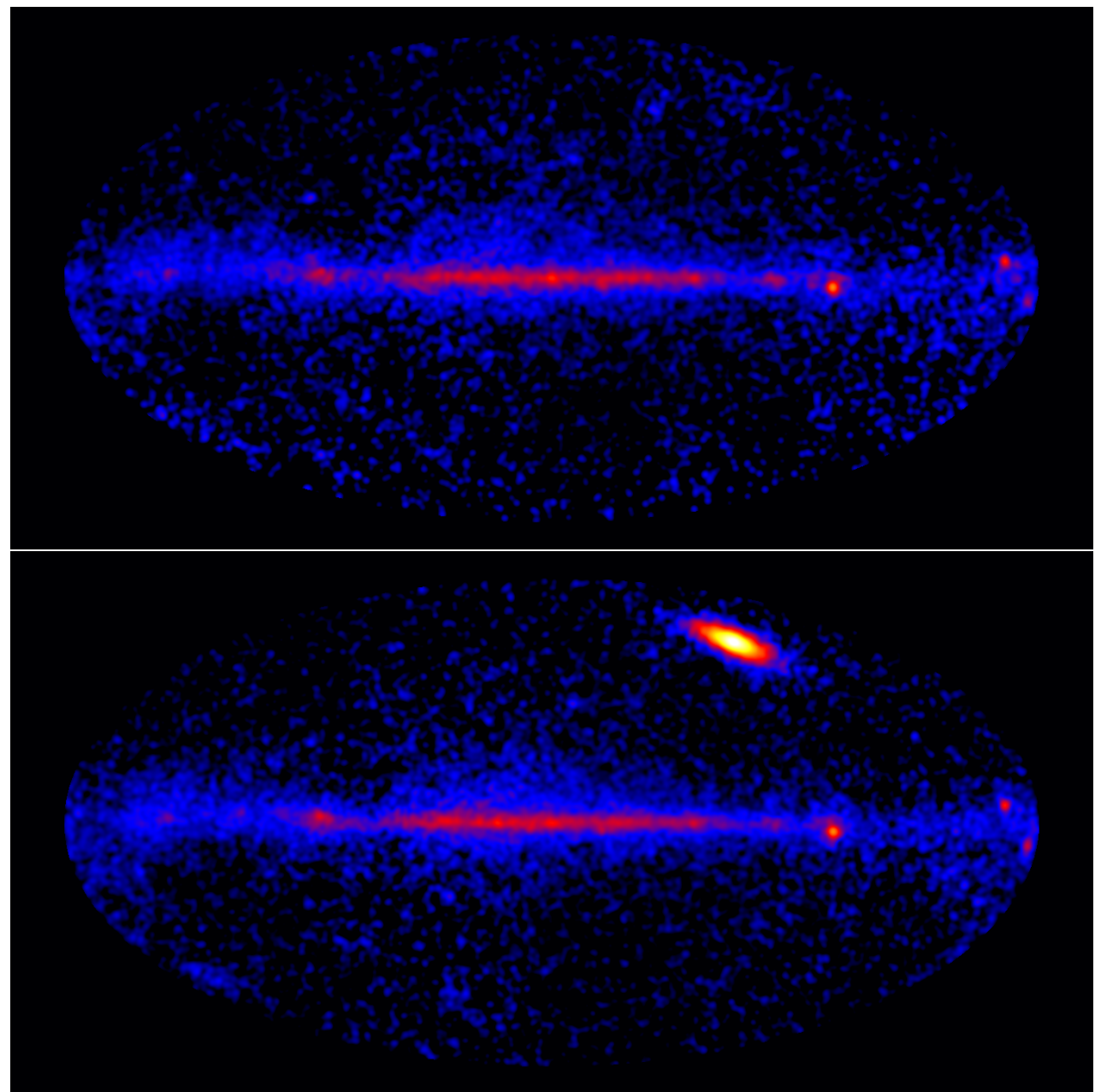

Figure 5: 12 hour intensity maps of the gamma-ray sky before (top panel) and during (bottom panel) the September 10, 2017 X8.2 solar flare. The flaring Sun was the brightest source in the sky during this flare. Long duration gamma-ray emission was detected by the LAT for 12 hours. The Fermi-LAT collaboration circulated an Astronomers Telegram (ATel 10721) on this flare.

the particles that produce the soft X-rays (emitted from the loop top), hard X-rays (emitted from the footpoints) and gamma-rays (emitted from the chromosphere near the footpoints). The long duration gamma-ray emission can originate from particles accelerated at the CME shock front which in turn precipitate back to the chromosphere to produce gamma-ray emission via pion decay. As the CME shock front moves away from the Sun, the particles accelerated precipitate further away from the active region to produce the gamma-rays and some of the particles from the same population escape from the CME shock front and are detected at Earth as solar energetic particles (as supported by [29]).

This scenario can also explain the observations of the BTL flares because the impulsive phase, where generally hard X-rays are emitted at the footpoints, is not detected while soft X-rays from the looptop and long-duration gamma-ray emission is detected. If the particles producing the gammaray emission are indeed accelerated at the CME shock and precipitate to the visible disk then the 
Fermi-LAT localization of the emission which points back to the visible disk would be in support of such a scenario.

In summary thanks to the Fermi-LAT observations of the Sun (both during its quiescent and the flaring state) we now have a better understanding of the acceleration mechanisms at work and how the Galactic cosmic-rays interact with the solar environment. The first Fermi-LAT solar flare catalog is in preparation as well as the time evolution and study of the two emission components from the quiescent Sun over the last 9 nine years.

\subsection{Questions asked}

Q: What is the highest energy photon detected from a solar flare?

A: The highest energy photons detected was the $4.5 \mathrm{GeV}$ photon coming from the solar flare of 2012 March 7. However we also have detected several hig energy photons from the 2017 September 10 flare. We are doing some cross checks and are working on a publication on this flare.

Q: Have you been able to detect motion in the localization as a function of time for any of the observed flares?

A: Yes, for the 2012 March 7 flare we performed a study of the the localization position as a function of time and found that indeed the position appeared move across the solar disk as time passed. This finding is in support of the emission scenario discussed during the presentation.

\section{Acknowledgments}

The Fermi LAT Collaboration acknowledges support from a number of agencies and institutes for both development and the operation of the LAT as well as scientific data analysis. These include NASA and DOE in the United States, CEA/Irfu and IN2P3/CNRS in France, ASI and INFN in Italy, MEXT, KEK, and JAXA in Japan, and the K. A. Wallenberg Foundation, the Swedish Research Council and the National Space Board in Sweden. Additional support from INAF in Italy and CNES in France for science analysis during the operations phase is also gratefully acknowledged.

\section{References}

[1] A. A. Atwood, W. B.Abdo, M. Ackermann, M. Ajello, L. Baldini, J. Ballet, G. Barbiellini et al., The Large Area Telescope on the Fermi Gamma-Ray Space Telescope Mission, apj 697 (June, 2009) 1071-1102, [0902.1089].

[2] D. J. Thompson, D. L. Bertsch, C. E. Fichtel, R. C. Hartman, R. Hofstadter, E. B. Hughes et al., Calibration of the Energetic Gamma-Ray Experiment Telescope (EGRET) for the Compton Gamma-Ray Observatory, apjs 86 (June, 1993) 629-656.

[3] D. J. Forrest, W. T. Vestrand, E. L. Chupp, E. Rieger, J. F. Cooper and G. H. Share, Neutral pion production in solar flares, in International Cosmic Ray Conference (M. Garcia-Munoz, K. R. Pyle, \& J. A. Simpson, ed.), vol. 4 of International Cosmic Ray Conference, pp. 146-149, Aug., 1985.

[4] S. Kuznetsov, V. Kurt, B. Yushkov, K. Kudela and V. Galkin, Gamma-ray and high-energy-neutron

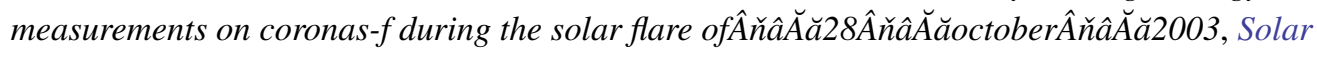
Physics 268 (2011) 175-193. 
[5] M. Ackermann, M. Ajello, A. Allafort, W. B. Atwood, L. Baldini, G. Barbiellini et al., Fermi Detection of $\gamma$-Ray Emission from the M2 Soft X-Ray Flare on 2010 June 12, apj 745 (Feb., 2012) 144.

[6] M. Ackermann, M. Ajello, A. Albert, A. Allafort, L. Baldini, G. Barbiellini et al., High-energy gamma-ray emission from solar flares: Summary of fermi large area telescope detections and analysis of two m-class flares, The Astrophysical Journal 787 (2014) 15.

[7] M. A. A. Ajello, A. Allafort, L. Baldini, G. Barbiellini, D. Bastieri, R. Bellazzini et al., Impulsive and long duration high-energy gamma-ray emission from the very bright 2012 march 7 solar flares, The Astrophysical Journal 789 (2014) 20.

[8] M. Pesce-Rollins, N. Omodei, V. Petrosian, W. Liu, F. Rubio da Costa, A. Allafort et al., First Detection of $>100 \mathrm{MeV}$ Gamma-Rays Associated with a Behind-the-limb Solar Flare, apjl 805 (June, 2015) L15, [1505.03480].

[9] M. Ackermann, A. Allafort, L. Baldini, G. Barbiellini, D. Bastieri, R. Bellazzini et al., Fermi-LAT Observations of High-energy Behind-the-limb Solar Flares, apj 835 (Feb., 2017) 219, [1702.00577].

[10] A. A. Abdo, M. Ackermann, M. Ajello, L. Baldini, J. Ballet, G. Barbiellini et al., Fermi Large Area Telescope Observations of Two Gamma-Ray Emission Components from the Quiescent Sun, apj 734 (June, 2011) 116.

[11] J. F. Dolan and G. G. Fazio, The gamma-ray spectrum of the sun, Reviews of Geophysics 3 (1965) 319-343.

[12] D. Seckel, T. Stanev and T. K. Gaisser, Signatures of cosmic-ray interactions on the solar surface, ApJ 382 (Dec., 1991) 652-666.

[13] I. V. Moskalenko, T. A. Porter and S. W. Digel, Inverse compton scattering on solar photons, heliospheric modulation, and neutrino astrophysics, The Astrophysical Journal Letters 652 (2006) L65.

[14] E. Orlando and A. W. Strong, Gamma rays from halos around stars and the sun, Astrophysics and Space Science 309 (Jun, 2007) 359-363.

[15] Orlando, E. and Strong, A. W., Gamma-ray emission from the solar halo and disk: a study with egret data, Astronomy and Astrophysics 480 (2008) 847-857.

[16] K. C. Y. Ng, J. F. Beacom, A. H. G. Peter and C. Rott, First observation of time variation in the solar-disk gamma-ray flux with Fermi, Physical Review D 94 (July, 2016) 023004, [1508. 06276 ].

[17] P. Hoyng, A. Duijveman, M. E. Machado, D. M. Rust, Z. Svestka, A. Boelee et al., Origin and Location of the Hard X-Ray Emission in a Two-Ribbon Flare, apjl 246 (June, 1981) L155.

[18] S. Masuda, T. Kosugi, H. Hara, S. Tsuneta and Y. Ogawara, A Loop-Top Hard X-Ray Source in a Compact Solar Flare as Evidence for Magnetic Reconnection, Nature 371 (Oct., 1994) 495.

[19] V. Petrosian, T. Q. Donaghy and J. M. McTiernan, Loop Top Hard X-Ray Emission in Solar Flares: Images and Statistics, apj 569 (Apr., 2002) 459-473, [astro-ph/0112363].

[20] L. Sui and G. D. Holman, Evidence for the Formation of a Large-Scale Current Sheet in a Solar Flare, apjl 596 (Oct., 2003) L251-L254.

[21] W. Liu, V. Petrosian, B. R. Dennis and Y. W. Jiang, Double Coronal Hard and Soft X-Ray Source Observed by RHESSI: Evidence for Magnetic Reconnection and Particle Acceleration in Solar Flares, apj 676 (Mar., 2008) 704-716, [arXiv:0 709.1963 ]. 
[22] W. Liu, Q. Chen and V. Petrosian, Plasmoid Ejections and Loop Contractions in an Eruptive M7.7 Solar Flare: Evidence of Particle Acceleration and Heating in Magnetic Reconnection Outflows, apj 767 (Apr., 2013) 168, [1303.3321].

[23] G. Kanbach, D. L. Bertsch, C. E. Fichtel, R. C. Hartman, S. D. Hunter, D. A. Kniffen et al., Detection of a long-duration solar gamma-ray flare on June 11, 1991 with EGRET on COMPTON-GRO, Astronomy and Astrophysics Supplement Series 97 (Jan., 1993) 349-353.

[24] W. T. Vestrand and D. J. Forrest, Evidence for a spatially extended component of gamma rays from solar flares, ApJL 409 (June, 1993) L69-L72.

[25] C. Barat, G. Trottet, N. Vilmer, J.-P. Dezalay, R. Talon, R. Sunyaev et al., Evidence for intense coronal prompt gamma-ray line emission from a solar flare, ApJL 425 (Apr., 1994) L109-L112.

[26] N. Vilmer, G. Trottet, C. Barat, R. A. Schwartz, S. Enome, A. Kuznetsov et al., Hard X-ray and gamma-ray observations of an electron dominated event associated with an occulted solar flare, Astronomy \& Astrophysics 342 (Feb., 1999) 575-582.

[27] E. W. Cliver, S. W. Kahler and et al., On the Origin of Gamma-Ray Emission from the Behind-the-Limb Flare on 29 September 1989, in International Cosmic Ray Conference, vol. 3 of International Cosmic Ray Conference, pp. 91-+, 1993.

[28] I. Plotnikov, A. Rouillard and G. Share, The magnetic connectivity of coronal shocks to the visible disk during long-duration gamma-ray flares, in EGU General Assembly Conference Abstracts, vol. 19 of EGU General Assembly Conference Abstracts, p. 4524, Apr., 2017, 1703.07563.

[29] G. A. de Nolfo, M. Boezio, A. Bruno, E. R. Christian, M. Martucci, M. Mergè et al., High-Energy Solar Energetic Particles \& Long Duration Gamma-Ray Flares - Is there a Connection?, AGU Fall Meeting Abstracts (Dec., 2016). 